BNL - 68013

\title{
Harmonic Inverse Free Electron Laser Micro-Buncher
}

\author{
S. Pottorf \\ Department of Physics \& Astronomy \\ State University of New York at Stony Brook \\ Stony Brook, New York 11794 \\ X.J. Wang \\ Brookhaven Accelerator Test Facility \\ National Synchrotron Light Source \\ Brookhaven National Laboratory \\ Upton, NY 11973, USA
}

October 2000

\section{National Synchrotron Light Source}

\author{
Brookhaven National Laboratory \\ Operated by \\ Brookhaven Science Associates \\ Upton, NY 11973
}

Under Contract with the United States Department of Energy

Contract Number DE-AC02-98CH10886 
DISCLAIMER

This report was prepared as an account of work sponsored by an agency of the United States Government. Neither the United States Government nor any agency thereof, nor any of their employees, nor any of their contractors, subcontractors or their employees, makes any warranty, express or implied, or assumes any legal liability or responsibility for the accuracy, completeness, or any third party's use or the results of such use of any information, apparatus, product, or process disclosed, or represents that its use would not infringe privately owned rights. Reference herein to any specific commercial product, process, or service by trade name, trademark, manufacturer, or otherwise, does not necessarily constitute or imply its endorsement, recommendation, or favoring by the United States Government or any agency thereof or its contractors or subcontractors. The views and opinions of authors expressed herein do not necessarily state or reflect those of the United States Government or any agency thereof. 


\title{
HARMONIC INVERSE FREE ELECTRON LASER MICRO-BUNCHER
}

\author{
S. POTTORF \\ Department of Physics \& Astronomy, State University of New York at Stony Brook \\ Stony Brook, NY 11794 \\ X.J. WANG \\ Brookhaven Accelerator Test Facility, NSLS, Brookhaven National Laboratory \\ Upton, NY 11973, USA \\ E-mail: xwang@bnl.gov
}

\begin{abstract}
The Inverse Free Electron (IFEL) and its applications are first briefly reviewed. The concept of harmonic IFEL was proposed for electron beam micro -bunching. A tightly micro-bunched electron beam could be used either as a laser accelerator injector or for femto -second X-ray generation by ultra-high harmonic radiation. For a planar undulator, the electron beam is strongly coupled to both the fundamental and odd harmonic if the undulator parameter is $\mathrm{g}$ reater than $1, \mathrm{~K}$ $>1$. The 1-D equation of motion for the IFEL was first extended to the third harmonic. 1-D simulations were performed for both fundamental only IFEL and harmonic IFEL. Two configurations of harmonic IFEL were considered. First, a single undulator with both fundamental and third harmonic IFEL presented simultaneously was studied. Second, a configuration employing a fundamental IFEL followed by a third harmonic IFEL. Better and efficient bunching was achieved for both harmonic IFEL comp ared to the fundamental IFEL only.
\end{abstract}

\section{Introduction}

The concept of the IFEL was proposed by Palmer [1] shortly after the free-electron laser (FEL) was re-introduced by Madey in 1971 [2]. The basic physics of the IFEL is very much similar to the FEL interaction. The undulator magnet introduces a small transverse velocity in the direction parallel to the electric vector of the electromagnetic wave. The interaction between the wave and electrons leads to an exchange of energy between the electron beam and wave. In the IFEL, the energy exchange leads to a loss of energy from the electromagnetic wave while the electromagnetic wave gains energy in the FEL. Extensive studies were carried out at Brookhaven National Laboratory [3,4] to explore the possibility of IFEL based electron accelerator. IFEL acceleration of an electron beam was observed at the Brookhaven Accelerator Test Facility (ATF) using a $10.6 \mu \mathrm{m} \mathrm{CO}_{2}$ laser [5].

The IFEL was also investigated as a UV or X-ray source with techniques such as laser seeded single-pass FEL [6] and high-gain harmonic radiation generation [7]. In the high-gain harmonic generation (HGHG) FEL [6], the interaction of the electron beam through the IFEL effect with a seed laser in the first undulator (modulator) leads to energy modulation of the electron beam. Energy modulation 
is transformed into spatial modulation (bunching) in the second HGHG magnet (dispersion section), and a micro-bunched electron beam generates coherent radiation and amplification in the last section of the HGHG undulator (radiator). More than seven orders of magnitude gain was reported at second harmonic of seed laser at the ATF HGHG experiment [6]. The main attractions of HGHG compared to the Self Amplified Spontaneous Emission (SASE) [8] are better longitudinal coherence and use of a shorter undulator and it suffers from the availability of seed laser. In the scheme of high harmonic radiation, IFEL interaction of the electron beam with a high power laser $(\mathrm{GW})$ leads to strong micro -bunching of the electron beam. Large harmonic contents of micro-bunched electron beam could produce coherent radiation through the second undulator in high harmonic radiation [7]. The IFEL effect was used to slice the femto-second electron beam out of picosecond long electron bunches in the ALS storage ring using a femto-second high power laser $[9,10]$. The IFEL also plays an important role in a proposed optical stochastic cooling technique [11].

IFEL micro-bunching with harmonic contents of the IFEL were experimentally observed at the ATF [12]. Furthermore, IFEL micro-bunched electron beams were captured and accelerated in a staged laser acceleration experiment [13]. The IFEL as an injector for laser accelerators has many merits. Since the IFEL interaction and bunching occur in vacuum, it only slightly degrades the quality of the electron beam. The same laser can be used both as a buncher and as an accelerator of an electron beam. The synchronization between electron micro -bunches and acceleration field is built in.

In both high harmonic generation and injection for laser accelerations, single stage IFEL bunching requires a high power laser, and efficiency is low. In the traditional RF linacs, those problems were overcome by using multi -stage harmonic bunchers. We are proposing a similar technique that could also be used as an IFEL. In a planar undulator with large undulator parameter, $K>1$, the electron motion in the undulator contains a fast longitudinal component, $\mathrm{k} \Delta \mathrm{z} \approx-$ $\xi \sin \left(2 \mathrm{k}_{0} \mathrm{z}\right)$, where $\xi=\mathrm{K}^{2} / 2\left(1+\mathrm{K}^{2}\right)$. This fast longitudinal motion leads to strong spontaneous emission at odd harmonics, $n=1,3,5 \ldots$ [14]. Similarly, the electron beam is also strongly coupled to odd harmonics in the IFEL interaction for planar undulators. Odd harmonic buncher cavities were employed in RF linacs because they generate a square wave form, therefore the harmonic IFEL can also be explored to improve electron beam micro -bunching.

In the following section, the derivation of 1-D IFEL equations (pendulum equations) for the fundamental frequen cy is reviewed. We then re-derive the equations of motion for two cases. First, we consider a third harmonic laser seed in the IFEL, and in the second case, the IFEL laser is composed of fundamental and third harmonics. In section III, we describe the simulations used to solve the 
1-D IFEL equations of motion, then we discuss the energy-phase space and energy and phase distribution results from the simulations.

\section{1-D Harmonic IFEL Theory}

Assuming an electron beam co -propagates with a laser along the undulator axis in the $\mathrm{z}$ direction, only longitudinal motion of the electrons will be considered. Following the basic formalism used in reference 3, the relativistic Hamiltonian is given by,

$$
H=\left[(\mathbf{P}-e \mathbf{A})^{2} c^{2}+m^{2} c^{4}\right]^{1 / 2}+e \Phi=\gamma m c^{2}+e \Phi
$$

where $\mathbf{P}=\mathbf{p}+e \mathbf{A}$ is the canonical momentum with $\mathbf{p}=\gamma m \mathbf{v}$ being the kinetic momentum and $\mathbf{A}$ the vector potential. The scalar potential $\Phi$ in the Hamiltonian is set to zero since space charge effects are not considered. The vector potential for a planar undulator is,

$$
\mathbf{A}_{w}=\hat{\mathbf{x}} \sqrt{2} A_{w} \sin \left(k_{w} z\right)
$$

where $k_{w}=2 \pi / \lambda_{w}$ with $\lambda_{w}$ is the undulator period. The magnetic field is,

$$
\mathbf{B}_{w}=\nabla \times \mathbf{A}_{w}=\hat{\mathbf{y}} k_{w} \sqrt{2} \cos \left(k_{w} z\right) .
$$

For $\mathbf{p}_{x} / \mathbf{p}_{z}<<1$, the motion due to the undulator alone is given by Hamilton's canonical momentum

$$
\begin{aligned}
& \mathbf{p}_{x}=-\hat{\mathbf{x}} e A_{w} \sqrt{2} \sin \left(k_{w} z\right) \\
& \mathbf{p}_{y}=0 \\
& \mathbf{p}_{z}=-\hat{\mathbf{x}} \gamma m c\left\{1-\frac{1}{2 \gamma^{2}}\left[1+K^{2}-K^{2} \cos \left(2 k_{w} z\right)\right]\right\}
\end{aligned}
$$

where $K$ is the undulator parameter,

$$
K=\frac{e B_{w} \lambda_{w}}{2 \pi m c} .
$$

The longitudinal motion of the electron can be written as,

$$
z=z_{o}+z^{\prime}
$$

where

$$
z_{o}=c t\left[1-\frac{1+K^{2}}{2 \gamma^{2}}\right]
$$

and its time derivative is 


$$
\frac{d z_{o}}{d t}=c\left[1-\frac{1+K^{2}}{2 \gamma^{2}}\right] .
$$

The small oscillations, $z^{\prime}$, are found by subtracting $d z_{o} / d t$ from $d z / d t$,

$$
\frac{d z^{\prime}}{d t}=\frac{K^{2} c}{2 \gamma^{2}} \cos \left[2 k_{w}\left(z_{o}+z^{\prime}\right)\right] \text {. }
$$

Expanding $z^{\prime}$ in powers of $1 / \gamma^{2}$, to the lowest order $z^{\prime}$ is

$$
z^{\prime}=\frac{K^{2} c}{4 \gamma^{2} k_{w}} \sin \left[2 k_{w}\left(z_{o}+z^{\prime}\right)\right] .
$$

The vector potential including the laser field is now given as

$$
\mathbf{A}=\hat{\mathbf{x}} \sqrt{2} A_{w} \sin \left(k_{w} z\right)+\hat{\mathbf{x}} \sqrt{2} A_{L} \sin \left(k_{L} z-\omega t+\phi_{L}\right) .
$$

Using Maxwell's equations, $\mathbf{E}=-\partial \mathbf{A} / \partial t$, gives us the electric field of the laser

$$
\mathbf{E}_{L}=\hat{\mathbf{x}} \sqrt{2} E_{L} \cos \left(k_{L} z-\omega t+\phi_{L}\right)
$$

where $E_{L} \approx \omega A_{L}, k_{L}=2 \pi / \lambda_{L}, \lambda_{L}, \omega$ are the laser amplitude, wavelength, and frequency. Note that we use a constant vector potential and initial phase $A_{L}$ and $\phi_{L}$ in the following equations.

The energy equation can be obtained from the time derivative of the Hamiltonian, $d H / d t=\partial H / \partial t$, to give

$$
\frac{d \gamma}{d t}=\frac{e}{\gamma m^{2} c^{2}} \mathbf{p}_{x} \bullet \mathbf{E}_{L}
$$

Substituting $\mathbf{p}_{x}$ and $\mathbf{E}_{L}$ into equation (13) we have

$$
\frac{d \gamma}{d t}=-\frac{e K E_{L}}{\gamma m c} 2 \sin \left(k_{w} z\right) \cos \left(k_{L} z-\omega t+\phi_{L}\right) .
$$

Eq. (14) can be rewritten as

$$
\frac{d \gamma}{d t}=-\frac{e K E_{L}}{\gamma m c}\left[\sin \left(\psi+\phi_{L}\right)-\sin \left(\psi+\phi_{L}-2 k_{w} z\right)\right] .
$$

Where $\psi=\left(k_{L}+k_{w}\right) z-\omega t$ is the electron phase. For the longitudinal position described by Eqs. $(6,7,8)$, the phase $\psi$ can be written, if the energy exchange is small for a single undulator period, as,

$$
\psi=\psi_{o}+\psi^{\prime}
$$

where 


$$
\psi_{o}=c t\left(k_{w}-k_{L} \frac{1+K^{2}}{2 \gamma^{2}}\right)
$$

and

$$
\psi^{\prime}=\frac{k_{L}}{k_{w}} \frac{K^{2}}{4 \gamma^{2}} \sin \left(2 k_{w} z_{o}\right)
$$

are the mean phase and small phase oscillations. The phase equation is then given by taking the time derivative of Eq. (17),

$$
\frac{d \psi_{o}}{d t}=c k_{w}\left(1-\frac{\gamma_{N}^{2}}{\gamma^{2}}\right)
$$

where $\gamma_{N}^{2}=\frac{k_{L}}{k_{w}} \frac{1+K^{2}}{2}$ is the resonance energy.

Substituting Eq. (16) into the energy equation, (15), we have

$$
\begin{aligned}
\frac{d \gamma}{d t}= & -\frac{e K E_{L}}{\gamma m c}\left\{\sin \left(\psi_{o}+\phi_{L}\right) \cos \psi^{\prime}+\cos \left(\psi_{o}+\phi_{L}\right) \sin \psi^{\prime}\right. \\
& \left.-\sin \left(\psi_{o}+\phi_{L}\right) \cos \left(\psi^{\prime}-2 k_{w} z\right)-\cos \left(\psi_{o}+\phi_{L}\right) \sin \left(\psi^{\prime}-2 k_{w} z\right)\right\}
\end{aligned}
$$

If $\gamma$ and $E_{L}$ change slowly (in our case $E_{L}$ is constant), then we can average Eq.(20) with respect to the mean position, $z_{o}$, over half a wiggler period,

$$
\begin{aligned}
\left\langle\frac{d \gamma}{d t}\right\rangle_{\theta}=- & \frac{e K E_{L}}{\gamma m c} \frac{1}{2 \pi}\left\{\sin \left(\psi_{o}+\phi_{L}\right) \int_{0}^{2 \pi} \cos (\xi \sin \theta) d \theta\right. \\
& +\cos \left(\psi_{o}+\phi_{L}\right) \int_{0}^{2 \pi} \sin (\xi \sin \theta) d \theta \\
& -\sin \left(\psi_{o}+\phi_{L}\right) \int_{0}^{2 \pi} \cos (\xi \sin \theta-\theta) d \theta \\
& \left.-\cos \left(\psi_{o}+\phi_{L}\right) \int_{0}^{2 \pi} \sin (\xi \sin \theta-\theta) d \theta\right\}
\end{aligned}
$$

where $\xi=\frac{k_{L} K^{2}}{k_{w} 4 \gamma^{2}}$, and $\theta=2 k_{w} z_{o}$, using the integral representation of the Bessel function given as, 


$$
J_{m}(u)=\frac{1}{2 \pi} \int_{0}^{2 \pi} \cos (u \sin \Theta-m \Theta) d \Theta
$$

where $m=0,1,2, \ldots$ Since sine functions average out to zero, we have

$$
\left\langle\frac{d \gamma}{d t}\right\rangle_{\theta} \rightarrow \frac{d \gamma}{d t}=-\frac{e K E_{L}}{\gamma m c} \sin \left(\psi_{o}+\phi_{L}\right)\left[J_{0}(\xi)-J_{1}(\xi)\right] .
$$

Making the independent variable to be $z$, using the approximation, $d z \approx c d t$, the 1-D IFEL equation is,

$$
\begin{gathered}
\frac{d \gamma}{d z}=-\frac{e K E_{L}}{\gamma m c^{2}} \sin \left(\psi_{o}+\phi_{L}\right)\left[J_{0}(\xi)-J_{1}(\xi)\right] \\
\frac{d \psi_{o}}{d z}=k_{w}\left(1-\frac{\gamma_{N}^{2}}{\gamma^{2}}\right) .
\end{gathered}
$$

For a $3^{\text {rd }}$ harmonic IFEL, the vector potential of the laser is

$$
\mathbf{A}=\hat{\mathbf{x}} \sqrt{2} A_{3} \sin \left[n\left(k_{L} z-\omega t\right)+\phi_{3}\right]
$$

where $n=3$ here. The electric field is then

$$
\mathbf{E}_{L}=\hat{\mathbf{x}} 3 \sqrt{2} E_{3} \sin \left[3\left(k_{L} z-\omega t\right)+\phi_{3}\right]
$$

where $E_{3} \approx \omega A_{3}$. Following the same procedure used in obtaining Eq. (24), we have,

$$
\begin{gathered}
\frac{d \gamma}{d z}=-\frac{3 e K E_{3}}{\gamma m c^{2}} \sin \left(3 \psi_{o}+\phi_{3}\right)\left[J_{1}(3 \xi)-J_{2}(3 \xi)\right] \\
\frac{d \psi_{o}}{d z}=k_{w}\left(1-\frac{\gamma_{N}^{2}}{\gamma^{2}}\right) .
\end{gathered}
$$

For an IFEL that uses a laser seed that includes up to the third harmonic gives equations of motion in 1-D as

$$
\begin{gathered}
\frac{d \gamma}{d z}=-\frac{e K}{\gamma m c^{2}}\left\{\begin{array}{l}
E_{1} \sin \left(\psi_{o}+\phi_{1}\right)\left[J_{0}(\xi)-J_{1}(\xi)\right]+ \\
3 E_{3} \sin \left(3 \psi_{o}+\phi_{3}\right)\left[J_{1}(3 \xi)-J_{2}(3 \xi)\right]
\end{array}\right\} \\
\frac{d \psi_{o}}{d z}=k_{w}\left(1-\frac{\gamma_{N}^{2}}{\gamma^{2}}\right)
\end{gathered}
$$




\section{1-D Simulation for harmonic IFEL and Discussion}

Table 1 lists the parameters used for our studies. The undulator parameter and beam energy are those used in the Stella experiment, so we used a Stella IFEL code to check our 1-D model for the fundamental laser seed only. IFEL Equations $(24,27,28)$ were solved using the Euler method of integration. The equations were converted to dimensionless units by introducing a unit length $z_{o}$,

$$
z_{o}=\frac{e E_{L}}{m c^{2}}
$$

where $E_{L}=\left(\mu_{o} / \varepsilon_{o}\right) I_{r}^{1 / 2}$ and $I_{r}=P_{L} / A$, where $I_{r}$ is the irradiance, $P_{L}$ is laser power, and $A$ is the cross-section of the laser. For the third harmonic only, $E_{3}=E_{L} / 3$. For the third and fundamental harmonics, $E_{L}$ is defined as

$$
E_{L}=E_{1}+E_{3}
$$

and $E_{3}=E_{1} / 3$ so that $E_{1}=(3 / 4) E_{L}$. The initial distribution of electrons is generated in such way that it is uniform in phase and Gaussian centered about the resonant energy (Fig.1). For a fixed laser power, the drift distance after the undulator was varied ( $20 \mathrm{~cm}$ to 1 meter) to optimize the bunching.

Table 1. Parameters for harmonic IFEL simulation.

\begin{tabular}{lll}
\hline & value & description \\
\hline$\lambda_{w}$ & $3.3 \mathrm{~cm}$ & Undulator period \\
\hline$\gamma_{N}$ & 85.76 & beam energy \\
$\lambda_{L}$ & 10.6 & $\begin{array}{c}\text { Fundamental IFEL } \\
\text { laser wavelength }\end{array}$ \\
$\mathrm{L}_{\mathrm{w}}$ & $26.4 \mathrm{~cm}$ & Undulator length \\
$\mathrm{K}$ & 1.93 & Undulator parameters \\
$\mathrm{PL}_{\mathrm{L}}$ & $150 \mathrm{MW}$ & Laser power \\
\hline
\end{tabular}



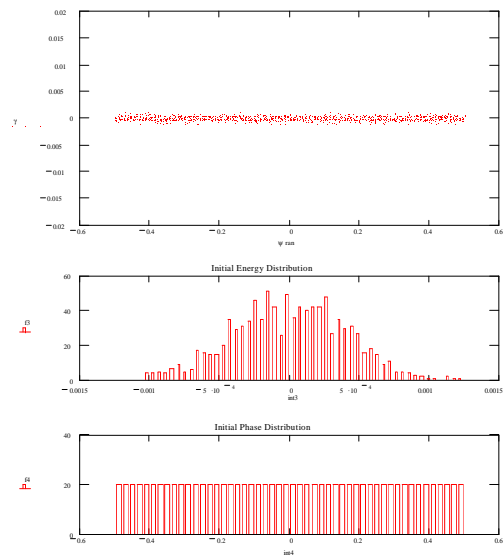

Figure 1. Electron Beam Initial distribution, phase space (top), energy spectrum (middle), bunch distribution (bottom).
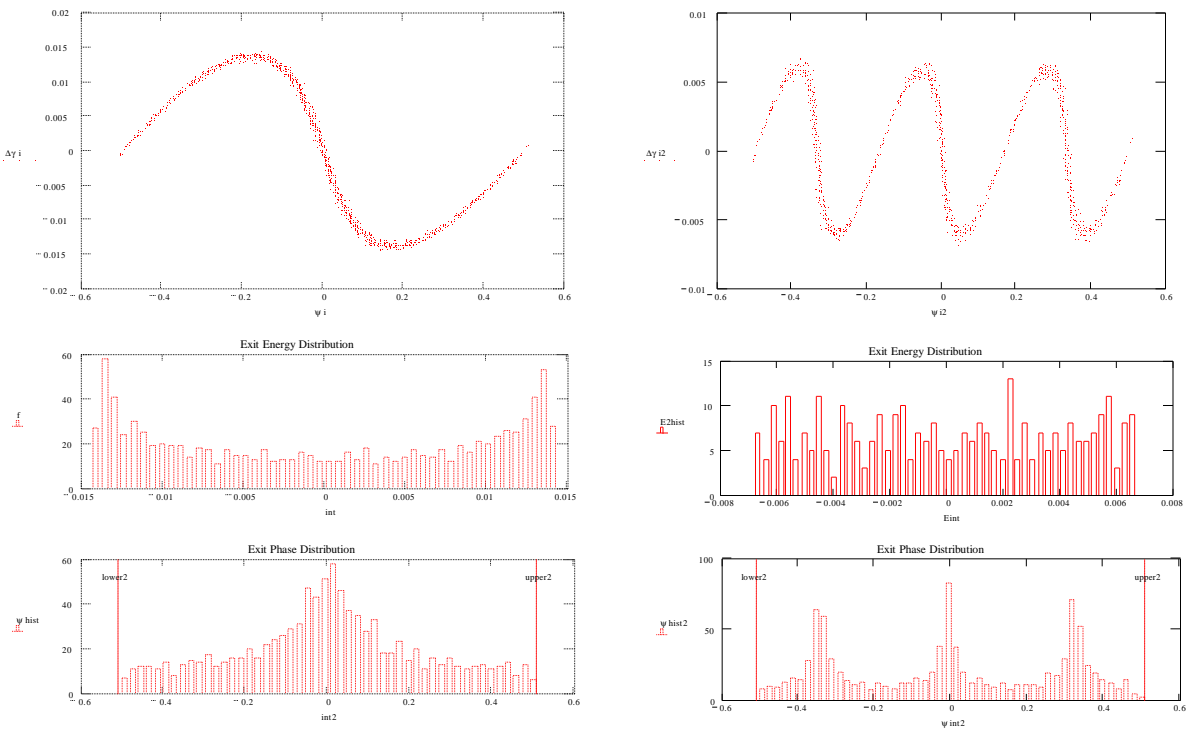

Figure 2. Electron Beam distributions after the fundamental IFEL (left) or third harmonic IFEL (right) using a single undulator. Phase space (top), energy spectrum (middle), bunch distribution (bottom). 

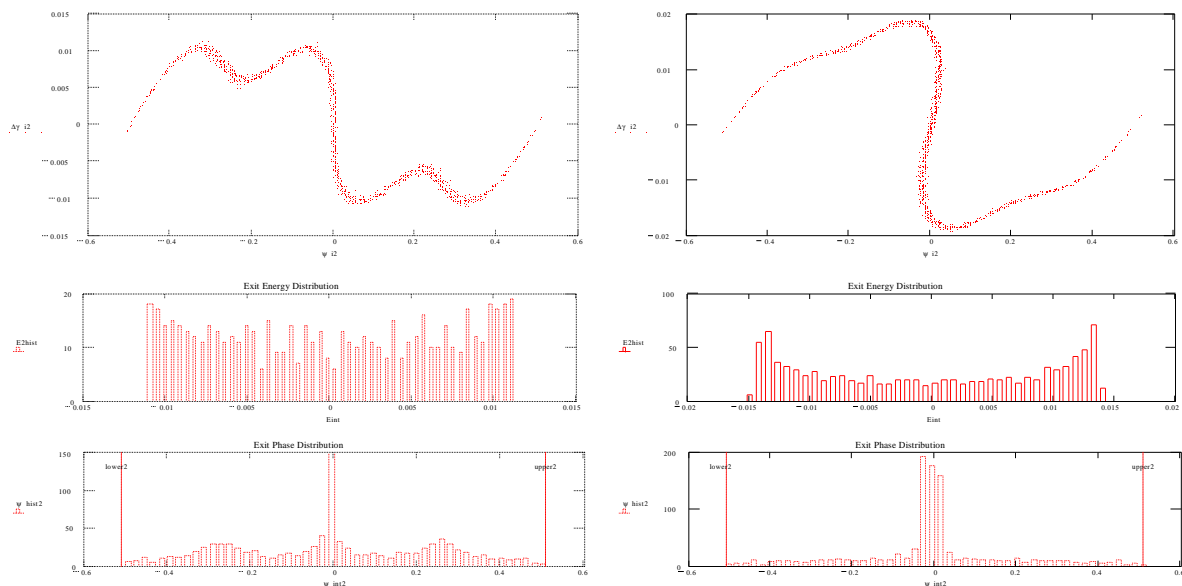

Figure 3. Electron beam distribution for $1^{\text {st }}$ and $3^{\text {rd }}$ harmonic IFEL (left). $1^{\text {st }}$ harmonic IFEL preceding a $3^{\text {rd }}$ harmonic IFEL (right). Phase space (top), energy spectrum (middle), bunch distribution (bottom).

Two configurations of the IFEL harmonic buncher were considered. In the first configuration, only one undulator is employed. In the second configuration, two undulators were employed. For the first configuration, single frequency interactions are shown in figure 2 for the fundamental (left) and third harmonic (right) IFEL. Also, in the first configuration, a combined frequency interaction of fundamental and third harmonics is shown in figure 3 (left). For the second configuration, single frequency interactions are used for each stage of the two undulators. Figure 3 (right) shows the beam distribution after using a fundamental IFEL for the first stage followed by a third harmonic IFEL for the second stage. The third harmonic laser power is about $10 \%$ of the total laser power for both configurations. Comparing fig. 2 and 3 we see that the combined harmonic IFEL and the staged IFEL improves the bunching by about one order of magnitude.

1-D simulations have shown significant improvement of electron beam micro bunching using a harmonic IFEL. We are now planning a third harmonic IFEL experiment at the BNL ATF using a $1 \mu \mathrm{m}$ photocathode RF gun driving laser. The second stage of the experiment will demonstrate harmonic IFEL bunching using both $1 \mu \mathrm{m}$ and its third harmonic in a single stage harmonic IFEL. We are also planning to inject a harmonic IFEL bunched electron beam into an IFEL accelerator to reduce the energy spread, then use the accelerated beam to study high gain ultra-high harmonic FEL. 


\section{Acknowledgements}

We would like to thank the ATF and Dr. I. Ben-Zvi for providing fund to the Stony Brook Research Foundation to support this study. The discussions with D.C. Quimby, W. D. Kimura of STI, and A. van Steenbergen are gratefully acknowledged. We would like also to thank D.C. Quimby and STI for making their IFEL computer program available for this study. This work is supported by US DOE under contract No. DE-AC02-98CH10886.

\section{References}

1. R. Palmer, J. Appl. Phys. 43, 3014(1972).

2. John M. Madey, J. Appl. Phys. 42, 1906 (1971).

3. E. D. Courant, C. Pellegrini, W. Zakowicz, Phys. Rev. A 32, 5, 2813 (1985).

4. A. Fisher, J. Gallardo, J. Sandweiss and A. van Steenbergen, Nucl. Instrum. Methods Phys. Res. Sect. A 341 ABS 111(1994).

5. A. van Steenbergen, J. Gallardo, J. Sandweiss, J. -M Fang, M. Babzien, X. Qiu, J. Skaritka and X.J. Wang, Phys. Rev. Lett. 77, 2690 (1996).

6. L.H. Yu et al, Science, 289, 932-936 (2000).

7. V.V. Goloviznin and P.W. van Amersfoot, Phys. Rev. E 55, 6002(1997).

8. R. Bonifacio, C. Pellegrini and L.M. Narducci, Opt. Commun. 50, 373 (1984).

9. R. W. Schoenlein et al, Science, 287, 2237-2240 (2000).

10. A.A. Zholents and M.S. Zolotorev, Phys. Rev. Lett. 76, 912 (1996).

11. A. Mikhalichenko and M. Zolotorev, Phys. Rev. Lett. 71, 4146 (1993).

12. Y. Liu, X.J. Wang, D. Cline, M. Babzien, J.M. Fang, J. Gallardo, K. Kusche, I. Pogorelsky, J. Skaritka, and A. van Steenbergen, Phys. Rev. Lett. 80, 20, 4418 (1998).

13. W.D. Kimura et al, "STELLA Experiment: Design and Model Predictions", BNL-66297 (1998).

14. W. B. Colson, IEEE J. Quantum Electron. QE-17, 1417 (1981).

ifel_qabp2kedited submitted to World Scientific 1/8/01 : 6:25 PM 